\title{
AUTOMATIC DETECTION OF CLOUDS AND SHADOWS USING HIGH RESOLUTION SATELLITE IMAGE TIME SERIES
}

\author{
Nicolas Champion \\ IGN-F, Ramonville Saint-Agne, France-nicolas.champion@ign.fr
}

Commission III, WG III/3

KEY WORDS: Clouds, Shadows, Landsat-8, Pléiades-HR, Time Series, Image Sequences

\begin{abstract}
:
Detecting clouds and their shadows is one of the primaries steps to perform when processing satellite images because they may alter the quality of some products such as large-area orthomosaics. The main goal of this paper is to present the automatic method developed at IGN-France for detecting clouds and shadows in a sequence of satellite images. In our work, surface reflectance orthoimages are used. They were processed from initial satellite images using a dedicated software. The cloud detection step consists of a region-growing algorithm. Seeds are firstly extracted. For that purpose and for each input ortho-image to process, we select the other ortho-images of the sequence that intersect it. The pixels of the input ortho-image are secondly labelled seeds if the difference of reflectance (in the blue channel) with overlapping ortho-images is bigger than a given threshold. Clouds are eventually delineated using a region-growing method based on a radiometric and homogeneity criterion. Regarding the shadow detection, our method is based on the idea that a shadow pixel is darker when comparing to the other images of the time series. The detection is basically composed of three steps. Firstly, we compute a synthetic ortho-image covering the whole study area. Its pixels have a value corresponding to the median value of all input reflectance ortho-images intersecting at that pixel location. Secondly, for each input ortho-image, a pixel is labelled shadows if the difference of reflectance (in the NIR channel) with the synthetic ortho-image is below a given threshold. Eventually, an optional region-growing step may be used to refine the results. Note that pixels labelled clouds during the cloud detection are not used for computing the median value in the first step; additionally, the NIR input data channel is used to perform the shadow detection, because it appeared to better discriminate shadow pixels. The method was tested on times series of Landsat 8 and Pléiades-HR images and our first experiments show the feasibility to automate the detection of shadows and clouds in satellite image sequences
\end{abstract}

\section{INTRODUCTION}

Detecting clouds and their shadows is one of the primary steps to perform when processing satellite images, especially during the computation of large cloud-free and shadow-free orthomosaics from input satellite images (Simpson and Stitt, 1998) (Irish e al., 2006).

On the one hand, the presence of clouds in input satellite images implies the acquisition of time series i.e. the acquisition of multi-temporal satellite images and computing large seamless orthomosaics from these multi-temporal images involves identifying cloud pixels in input data so that only cloud-free pixels are used to generate the final product (Din-Chang et al., 2008)

On the other hand, the shadows (of clouds) may cause some problems in the procedure, especially during the radiometric equalization. In particular, they may cause a "leopard skin" effect in the final orthomosaics (Soille, 2008). As a consequence, these shadows must also be detected so that another shadow-free image in the sequence is used to compute the final orthomosaics.

In (Champion, 2012), we introduced a method for detecting clouds in a Spot 5-HRS time series. The principles of this method will be explained later in this paper (Section3). The first step of this method consisted in a radiometric pre-processing step. Following the recommendations found in (Lillesand et al., 2008), the idea was to adapt the dynamic of input images by linearly stretching histogram between min (0) and max (255) values.
Good results were achieved using this method, as illustrated in Figure 1. When tested on a sequence of Pléiades-HR images, our experiments showed that the previously-described radiometric correction was not sophisticated enough to harmonize the radiometric aspects of input images and to ensure the stability of the detection, as illustrated in Figure 2.

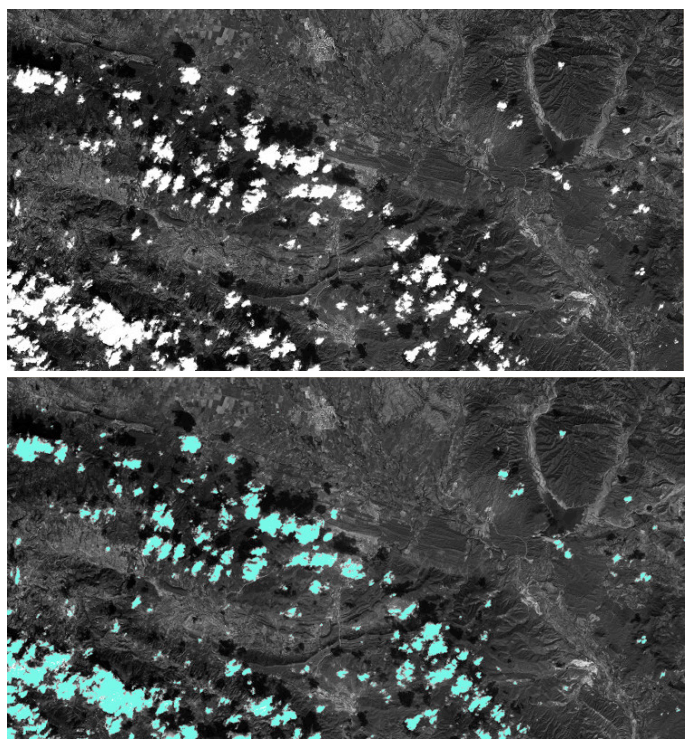

Figure 1. (top) Initial panchromatic SPOT5-HRS ortho-image. (bottom) The outcomes of our cloud detection algorithm (pixels labelled clouds in blue). 


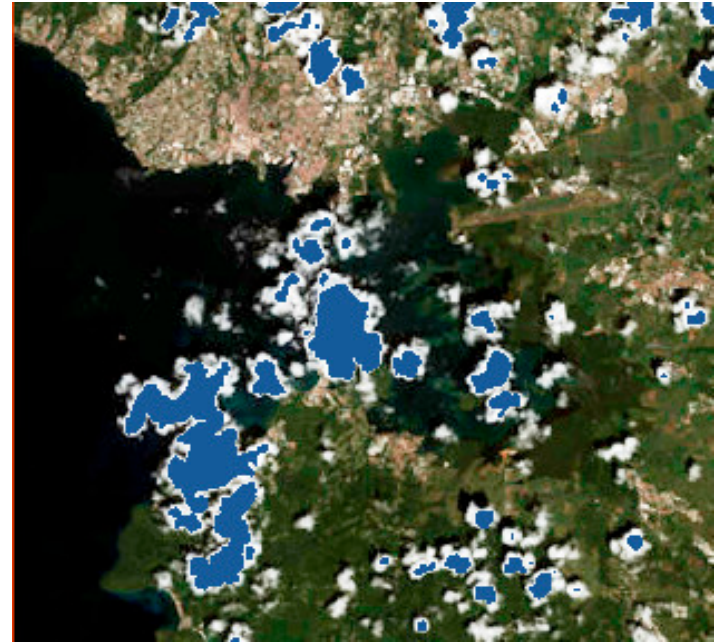

Figure 2. In blue, pixels detected as clouds on a Pléiades-HR image during the test, carried out in 2012. Some clouds are not detected by the first version of our method.

The main goals of this paper are firstly to present the improvements we brought to the initial procedure in order to make it usable with Pléiades-HR images, secondly to describe the method used to detect shadows. In particular, we will see that the use of reflectance images enables to improve the cloud and shadow detection greatly.

The paper is organized as follows. Section 2 describes input data; Section 3 details the methods used both for the cloud and the shadow detection; Section 4 presents the outcomes of our method and illustrates comparisons with existing cloud masks; Eventually, Section 5 draws conclusions and perspectives.

\section{INPUT DATA}

Two different kinds of input data are considered in our experiments: multispectral Landsat 8 ortho-images and 4 channels Pléiades-HR ortho-images. Note that, in the rest of the paper, we will use the terms image an ortho-image indifferently.

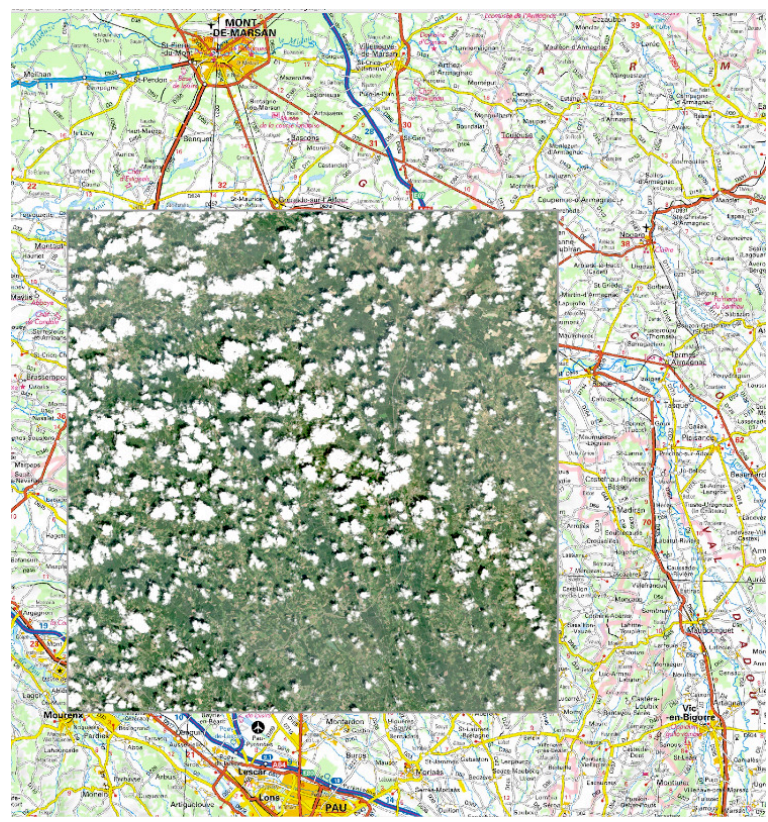

Figure 3. Initial Landsat 8 ortho-image (depicted here in a RGB mode) and its localisation in southern France.
Landsat 8 data. The first data set is composed of 72 Landsat 8 images. They have a spatial resolution of $30 \mathrm{~m}$ and 7 spectral bands: coastal aerosol, blue, green, red, near infra-red and two shortwave infra-red. The test area is located in Southern France and covers an area of about $2000 \mathrm{~km} 2$. Images were acquired between April 2013 and January 2016.

Pléiades-HR images. The second data set is composed of 9 Pléiades-HR (PHR) images. They have a spatial resolution of $50 \mathrm{~cm}$ and 4 spectral bands: RGB and near infra-red. The test area is located in Martinique, French West Indies and covers an area of about $150 \mathrm{~km}^{2}$. Images were acquired between March 2013 and April 2014.

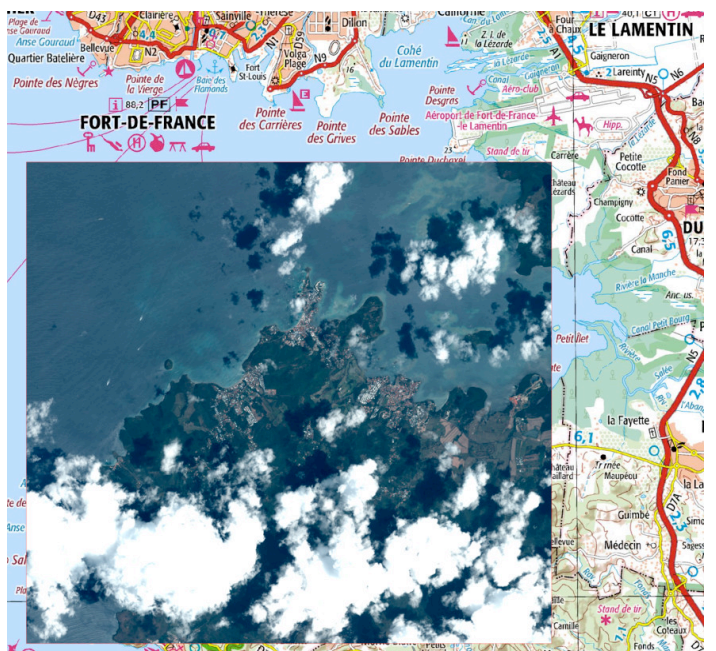

Figure 4. Initial Pléiades-HR ortho-image (depicted here in a RGB mode) and its localisation in the French West Indies.

\section{METHOD}

Compared to (Champion, 2012), the novelty of the method presented in this paper is related to the preliminary computation of reflectance images.

Regarding Landsat 8 images, TOC $^{1}$ reflectance images, corrected from the DTM effects, were computed from the initial satellite images using the MACCS software (Hagolle et al., 2015).

Regarding Pléiades-HR images, we used an in-house algorithm, based on the radiative transfer code 6S (Vermote et al., 1997) and the acquisition information available with the input satellite image (within the so-called DIMAP file) in order to convert the input digital accounts of PHR images into $\mathrm{TOA}^{2}$ reflectance values, including atmospheric corrections. More details can be found in (Le Hir et al., 2016).

In addition to these atmospheric corrections, Pléiades-HR images were downsampled to $20 \mathrm{~m}$ because the objects to be detected are big and do not require full resolution image processing. For Landsat 8 time series, the resolution of input images $(30 \mathrm{~m})$ is used.

\subsection{Cloud detection}

The method used in this paper to detect clouds is very similar to the one described in (Champion, 2012) and appears to be a compilation of the ideas found in (Hagolle et al., 2010), (Sedano et al., 2011) and (Le Hégarat-Mascle and André, 2009).

\footnotetext{
${ }^{1}$ TOC for Top of Canopy

${ }^{2}$ TOA for Top of Atmosphere
} 
Firstly, seeds are extracted. For that purpose, we take one orthoimage as a reference (this Reference Ortho-image RO corresponds to the ortho-image we want to detect clouds in). Secondly, we select the other ortho-images of the sequence that intersect this Reference Ortho-image RO and we crop them according to their overlapping on the Reference Ortho-image. From there, a pixel-to-pixel analysis is carried out between the Reference Ortho-image RO and each PO. If the radiometric difference is better than a given threshold $T_{R}$, the pixel of the Reference Ortho-image receives a positive vote. After the process being carried out for each $\mathrm{PO}$, a pixel of RO is labelled cloud if it receives one vote at least (in the previous version of the method, a majority of $2 / 3$ was required). Note that a regiongrowing algorithm may be used to refine results. It is based on a radiometric criterion and a homogeneity criterion. To be aggregated to a cloud region, the radiometry of the pixel must also lie within a range of given values and all the neighbours of this pixel must satisfy this criterion. In the experiments presented here, it was not necessary to perform this step because the sequence was large enough (72 images for Landsat 8 and 9 for PHR). Eventually, a morphological closing and opening are performed to eliminate residual noise.

To be totally exhaustive, we need to specify that median reflectance images (computed with a size of 2 pixels for the structuring element) were used instead of input reflectance PHR images. The aim here was to eliminate bright objects (e.g. large white buildings) that may be confused with clouds.
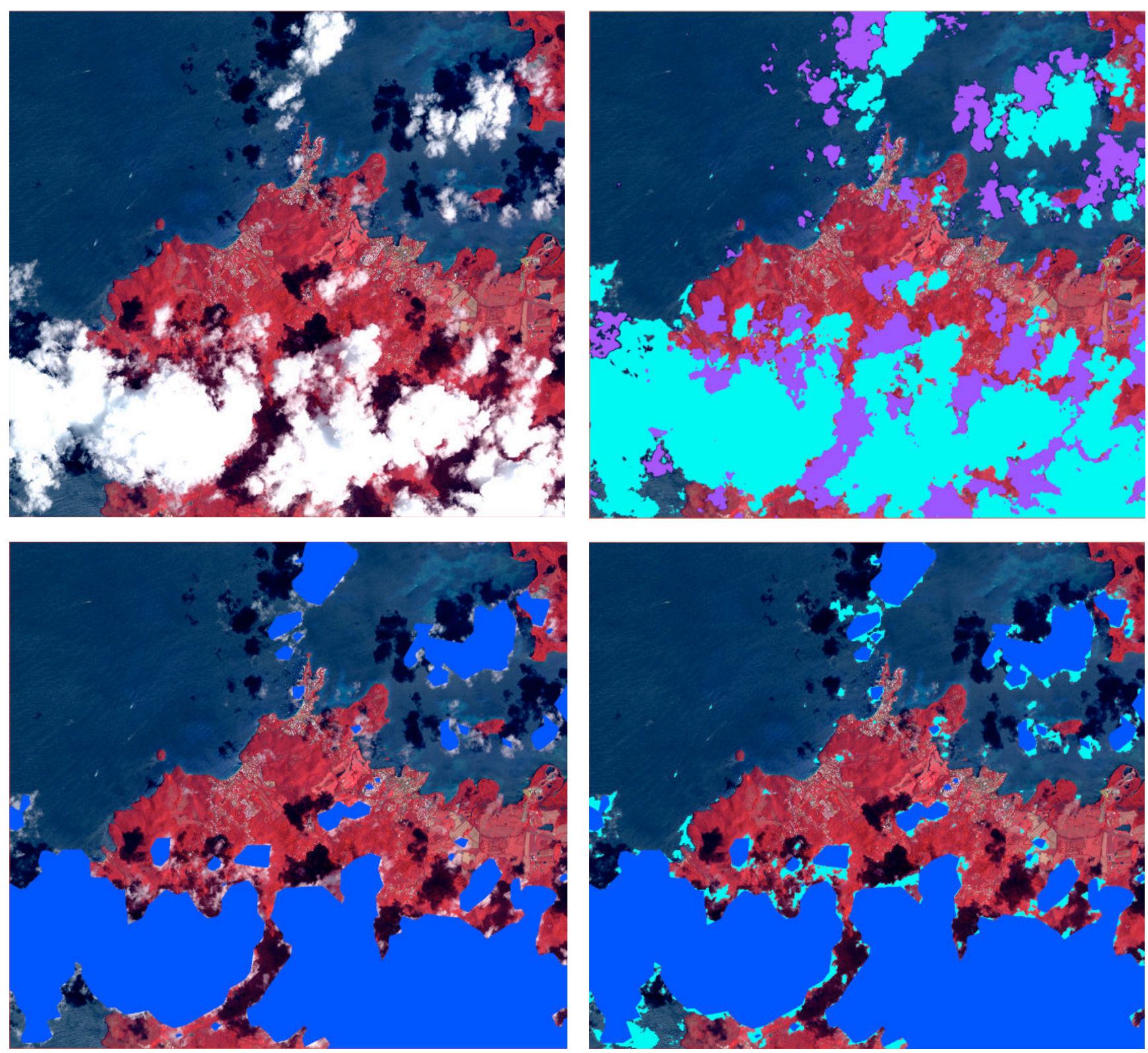

Figure 5. (top left) Initial Pléiades-HR image, depicted here in a CIR mode. (top right) the outcomes of our method; in cyan, the detection of clouds and in violet, the detection of shadows. (bottom left) The existing cloud mask, provided with input Pléiades-HR images. (bottom right) The existing cloud mask (in blue), superimposed on our cloud mask (in cyan). 

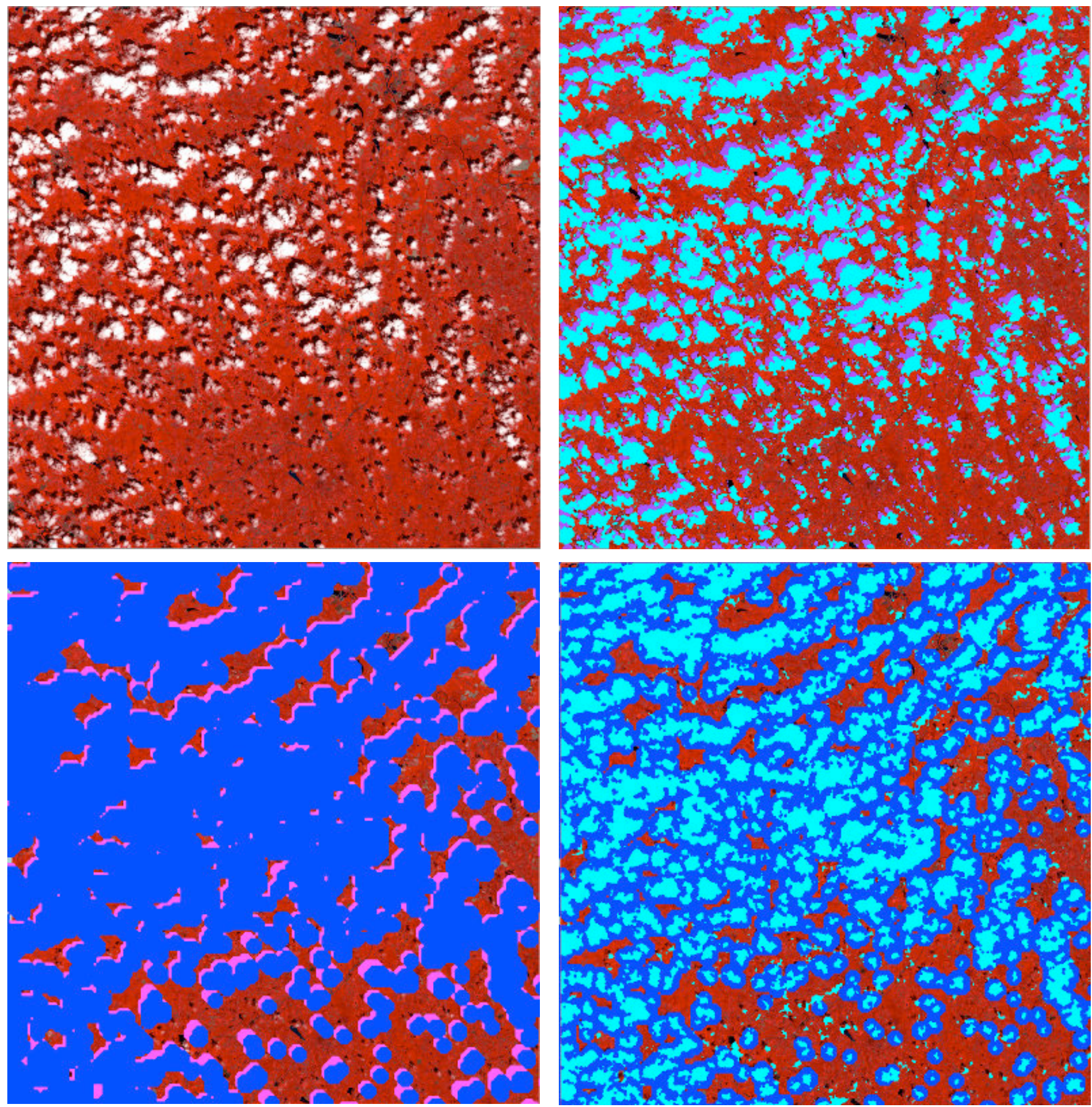

Figure 6. (top left) Initial Landsat 8 image, depicted here in a CIR mode. (top right) the outcomes of our method; in cyan, the detection of clouds and in violet, the detection of shadows. (bottom left) The existing cloud mask (in blue) and shadow mask (in purple), provided with input Landsat 8 images and computed using the MACCS software. (bottom right) The existing cloud mask (in blue), superimposed on our cloud mask (in cyan).

\subsection{Shadow detection}

The procedure to compute shadow masks is based on the idea by (Rosin and Ellis, 1995) that a shadow pixel is darker when comparing to the other images of the time series. This detection is basically composed of three steps.

Firstly, we compute a synthetic ortho-image covering the whole study area. Its pixels have a value corresponding to the median value of all input ortho-images intersecting at that pixel location. Secondly, for each input ortho-image, a pixel is labelled shadows if the difference of reflectance (in the NIR channel) with the synthetic ortho-image is below a given threshold. Eventually, a morphological closing is performed to refine the outcomes.

Note that pixels labelled as clouds in the first step are not used for computing the median value in the first step. In addition, the NIR input data channel is used to perform the shadow detection because it appeared to better discriminate shadows, as shown in (Richter and Mueller, 2005). Eventually, the PHR test zone contain sea areas. Even if the shadow detection is useful only on the ground (in our context), the procedure was adapted to work in marine areas. For that purpose, the synthetic image was 
computed using a $20 \%$ median (instead of a classic median) and a different value for thresholding images of difference was used.

\section{RESULTS AND DISCUSSION}

Regarding the cloud detection, the outcomes of our method are presented in Figures 5 and 6, and appear to be good. More specifically, we can observe that most of clouds are correctly detected by our method, both in the Landsat 8 and Pléiades-HR images. Comparing our outcomes with the cloud masks provided with Landsat 8 data (and computed using the MACCS software) and those provided with Pléiades-HR input products, it can be observed that the accuracy and granularity of our product are better, as depicted in Figure 5 (bottom right) for PHR images and Figure 6 (bottom right) for Landsat 8 images. Regarding the shadow detection, the outcomes obtained with Landsat 8 time series are good, as illustrated in Figure 6 (top right). In addition, the algorithm appears to be stable from one image to another. This is not the case for Pléiades-HR. Our experiments show the feasibility to compute shadow masks from PHR sequence but they also show that the algorithm is not stable.

If we push the analysis further, we can see that the atmospheric corrections computed with our in-house method and applied to PHR sequences are not satisfying in the NIR channel. More precisely, the time series for the NIR reflectance does not appear to be stable, contrary to what we observe with the Landsat 8 NIR reflectance sequences. This may be due to the parameterization of the $6 \mathrm{~S}$ software used in our work. In the future, we will use another tool, namely the optical calibration tool available in the Orfeo Tool $\mathrm{Box}^{3}$, to produce Pléiades-HR TOC images. The first tests carried out in that sense show real improvements.

\section{CONCLUSIONS AND PERSPECTIVES}

In this paper, we have presented an improved version of a method designed for detecting clouds in a sequence of satellite images. Our experiments have shown the stability of the method.

We have also introduced a method for detecting the shadows of clouds. Our experiments have shown that the reproducibility of the outcomes was depending on the stability of the reflectance sequences in the NIR channel. The Landsat 8 sequence, computed with the MACCS software, ensures this stability. But this is not the case for Pléiades-HR sequences.

In a near future, we plan to integrate the TOC computing functionality available in OTB to make our method more robust. In addition, we plan to test and adapt an in-house algorithm (named Mosar) in order to produce large cloud-free and shadow-free orthomosaics, starting from PHR sequences and the cloud and shadow masks presented here.

\section{REFERENCES}

Champion, N., 2012. Automatic cloud detection from multitemporal satellite images: towards the use of Pléiades time

${ }^{3}$ https://www.orfeo-toolbox.org - Last Visited: 2016-04-01. The OTB project was initiated and funded by CNES (French space agency) in the frame of a program named ORFEO to prepare, accompany and promote the use and the exploitation of the images derived from Pléiades-HR satellites. series. In: International Archives of the Photogrammetry, Remote Sensing and Spatial Information Sciences, Vol. B3.

Din-Chang, T., Hsiao-Ting, T., Chun-Liang, C., 2008. Automatic cloud removal from multi-temporal SPOT images. In: Applied Mathematics and Computation, Vol. 205(2), pp. 584-600.

Hagolle, O., Huc, M., Villa Pascual, D., Dedieu, G., 2010. A multi-temporal method for cloud detection, applied to FORMOSAT-2, VEN $\mu$ S, LANDSAT and SENTINEL-2 images. In: Remote Sensing of Environment, Vol. 114(8), pp. $1747-1755$.

Hagolle, O.Huc, M., Villa Pascual, D., Dedieu, G., 2015. A Multi-Temporal and Multi-Spectral Method to Estimate Aerosol Optical Thickness over Land, for the Atmospheric Correction of FormoSat-2, LandSat, VEN $\mu \mathrm{S}$ and Sentinel-2 Images. In: Remote Sensing 7 (3).

Irish, R.R., Barker, J.L., Goward, S.N., Arvidson, T., 2006. Characterization of the landsat-7 ETM+ automated cloud-cover assessment (ACCA) algorithm. In: Photogrammetric Engineering and Remote Sensing, Vol. 72(10), pp. 1179-1188.

Le Hégarat-Mascle, S., André, C., 2009. Use of Markov Random Fields for automatic cloud/shadow detection on high resolution optical images. In: International Journal of Photogrammetry and Remote Sensing, Vol. 64(4), pp. 351-366.

Le Hir, E., Massera, S., Favé, P., Lacherade, S. 2016. Physical Normalization of Spot 6/7 images. paper in preparation.

Lillesand, T. M., Kiefer, R.W. Chipman, J. W., Hoboken, N. J., 2008. Remote sensing and image interpretation. John Wiley \& Sons.

Richter, R., Mueller, A., 2005. De-shadowing of satellite/airborne imagery. In: International journal of Remote Sensing, Vol. 26, pp. 3137-3148.

Rosin, P. L. and Ellis, T. J., 1995. Image difference threshold strategies and shadow detection. In: Proceedings of the British Machine Vision Conference (BMVC).

Sedano, F., Kempeneers, P., Strobl, P., Kucera, J., Vogt, P., Seeabach, L., San-Miguel-Ayanz, J., 2011. A cloud mask methodology for high-resolution remote sensing data combining information from high and medium resolution optical sensors. In: International Journal of Photogrammetry and Remote Sensing, Vol 66(5).

Simpson, J. J., Stitt, J. R., 1998. A procedure for the detection and removal of cloud shadow from AVHRR data over land. In: IEEE Transaction on Geoscience and Remote Sensing. Vol. 36(3), p. 880-897.

Soille, P., 2008. IMAGE-2006 Mosaic: Cloud detection on SPOT-4 HRVIR, SPOT-5 HRG, and IRS-LISS III. Technical report, European Commission, Joint Research Centre

Vermote, E.F., Tanré, D ., Deuzé, J.L., Herman, M., Morcette, J.J., 1997. Second simulation of the satellite signal in the solar spectrum, 6S: an overview. IEEE Transactions on Geoscience and Remote Sensing, 3 5(3), pp. 675-686. 